\title{
37-40GHz MMIC Sub-Harmonically Pumped Image Rejection Diode Mixer
}

\author{
F. Rasà *, F. Celestino *, M. Remonti *, B. Gabbrielli *, P. Quentin ** \\ * ALCATEL ITALIA, TSD-HCMW R\&D, Str. Provinciale per Monza, 33, 20049 Concorezzo (MI) - ITALY \\ ** UNITED MONOLITHIC SEMICONDUCTORS, RD128, BP46, 91401 Orsay, Cedex -FRANCE \\ francesco.rasa@netit.alcatel.it francesco.celestino@netit.alcatel.it marcodaniele.remonti@netit.alcatel.it \\ barbara.gabbrielli@netit.alcatel.it pierre.quentin@ums-gaas.com
}

\begin{abstract}
The design simulations of a subharmonically pumped image rejection diode mixer for $K_{u}$ band operation is described. Details of design approach and outcome of performance simulations will be presented showing, in the $37 \div 40 \mathrm{GHz} \mathrm{RF}$ band with $2.5 \mathrm{GHz}$ of IF frequency, a typical conversion loss $\leq 12.5 \mathrm{~dB}$ with a $2 \mathrm{LO} / \mathrm{RF}$ isolation $\geq 70 \mathrm{~dB}$ and an image rejection $\geq 20 \mathrm{~dB}$ along with an $I_{3} \geq 1 \mathrm{dBm}$, for an LO drive power level of $+\mathbf{1 2 d B m}$, for a final compact MMIC size of $2.37 \times 2.31 \mathrm{~mm}^{2}$.
\end{abstract}

\section{INTRODUCTION}

This work, as a part of a co-operation plan between Alcatel Italy, Thomson-CSF Microelectronic and United Monolithic Semiconductors (UMS), moves towards the development of typical goals of GaAsbased MMIC technology to be employed in a new $38 \mathrm{GHz}$ microwave module for Urban Radio Systems to meet the increasing demand for low cost, easy deployable high capacity transmission systems.

The new family of transceiver, using a 128QAM modulation, require high linearity and high output power performances. In a typical transmitter chain, the mixer at the input of the module, must satisfy challenging $3^{\text {rd }}$ order intermodulation distortion performance and for broad-band operation inherent isolation property too. Typical fundamental diode mixes should operate at low output power level thus making difficult to obtain an adequate LO suppression. One could think of reducing the LO pumping power level, but this would seriously damage the frequency conversion efficiency and the linearity.

Therefore, an intrinsically LO-dumping circuit architecture is necessary, and a sub-harmonically pumped (SHP) mixer becomes an attractive solution.

SHP diode mixer is classically implemented feeding LO signal to a couple of anti-parallel diodes, the resulting combined conductance waveform has only even harmonic components, which means the converted RF products are placed around the 2LO frequency with inherently smaller residual power at twice the local oscillator frequency [1,2]. Moreover, since the LO frequency is half of the frequency needed in conventional fundamentally-pumped mixer, the SHP architecture offers the advantage of area reduction and system simplification, especially when working at high frequency, that is final cost reduction.

The RF image rejection (IMR) filtering is not feasible for broad-band operation, thus making intrinsic imagecutting circuit necessary, which is easily accomplished with a balanced two SHP mixer with quadrature input/output hybrids.

In this paper, the design and simulation performances of an MMIC $K_{u}$ sub-harmonically pumped image rejection (SHPIMR) diode mixer with an LO buffer amplifier are shown.

\section{DESIGN APPROACH}

The SHP mixer schematic used in this design is shown in figure 1.

At the output diode pair side, an open one-quarter wavelength long stub at LO frequency grounds the LO signal. On the other diode pair side, a shorted onequarter wavelength long stub at LO frequency grounds the 2LO, or approximately the RF signal. The input stub also provides an IF return path. A capacitor on the RF port blocks the IF leakage, while a double one-quarter wavelength long stubs at RF frequency avoids the RF leakage through the IF port. A DC de-coupling capacitor is placed at the LO port. No dc return path is necessary for the anti-parallel diode pair.

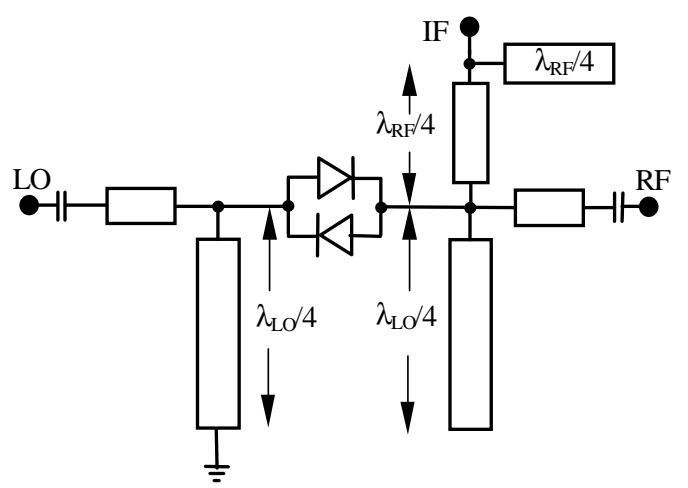

Fig.1 - Schematic view of SHP diode mixer

The needed image-rejection behaviour is achieved arranging two identical SHP mixers in a symmetrical quadrature structure, as illustrated in figure 2. 


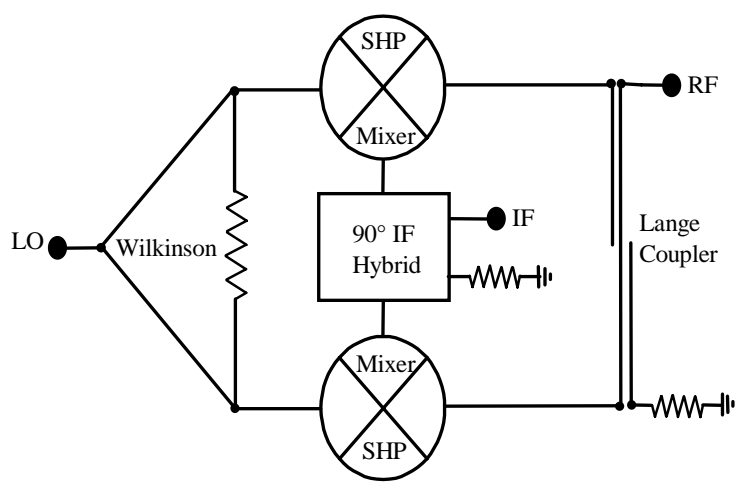

Fig.2 - SHPIMR Image-rejection mixer

The local oscillator pump is fed in-phase to the two SHP mixers via a Wilkinson power splitter; two IF signals coming from a $90^{\circ}$ IF hybrid coupler are then up-converted to RF and then combined by a $90^{\circ}$ Lange coupler that produces the desired image rejection at the RF output. The upper/lower side band (USB/LSB) cases are selected by choosing the right input of IF hybrid while the other port is terminated on $50 \Omega$. Due to the IF low frequency to be used, the IF hybrid is not included on the MMIC, not to exceed the area occupation constraints, thus resulting in two on chip input IF ports to be fed in quadrature.

The SHPIMR diode mixer MMIC also provides an LO buffer amplifier stage in order to fix the LO pump to the required diode input level and ensuring adequate isolation of input port. To avoid buffer-generated harmonics compromising mixer $2 \mathrm{LO}$ carrier suppression, a lumped low-pass section is inserted between the buffer and SHPIMR mixer.

\section{DESIGN IMPLEMENTATION AND PERFORMANCE SIMULATION}

The frequency specification for the mixer consists of an RF frequency in the $37 \div 40 \mathrm{GHz}$ band, an IF frequency up to $2.5 \mathrm{GHz}$ thus falling the $\mathrm{LO}$ signal in the range of $17.25 \div 21.25 \mathrm{GHz}$. The GaAs-based PH25 UMS process, provides both reliable diode and active device working at $\mathrm{K} / \mathrm{K}_{\mathrm{u}}$-band. Based upon a $0.25 \mu \mathrm{m}$ T-shape aluminium gate PHEMT MBE technology, this foundry process exploits an $\mathrm{f}_{\mathrm{t}}=70 \mathrm{GHz}, 330 \mathrm{pF} / \mathrm{mm}^{2}$ silicon nitride MIM capacitors, $120 \Omega / \mathrm{sq} \mathrm{GaAs,} \mathrm{30 \Omega /sq} \mathrm{TaN}$ and $1000 \Omega /$ sq TiWSi resistors, spiral square inductors, air bridge and via holes; supplying all the elements needed for this project. Two low-junction series resistance $4 \times 10 \mu \mathrm{m}$ diodes were used for the anti-parallel pair in the mixer core and a $8 \times 75 \mu \mathrm{m}$ wide PHEMT was selected for the buffer amplifier stage; active and lumped passive elements were modelled by means of the $\mathrm{PH} 25$ process library [3] and simulated by the
Agilent/EEsof Series IV software tool. The Momentum electromagnetic simulator was weightily used to solve discontinuity and coupling problems.

\section{A. SHP Mixer Design}

The SHP mixer design required attention on the symmetry of layout connection of the diodes pair, on the size and the relative placement of one-quarter wavelength stubs, on lines connection and on discontinuity evaluation. The simulations and the electromagnetic optimisations performed, lead to a circuit that in the $37 \div 40 \mathrm{GHz} \mathrm{RF}$ band shows close to $12.5 \mathrm{~dB}$ of conversion loss and high $2 \mathrm{LO}$ isolation, with an input LO power of $+16 \mathrm{dBm}$ in the $17.25 \div 21.25 \mathrm{GHz}$ band and an IF power of $-15 \mathrm{dBm}$ at $2.5 \mathrm{GHz}$, as shown in figure 3 and figure 4.

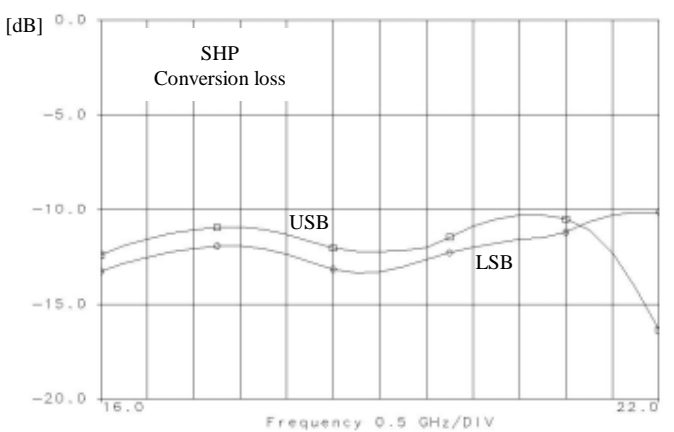

Fig. 3 - SHP mixer USB and LSB conversion losses $\mathrm{LO}+16 \mathrm{dBm}, \mathrm{IF}=-15 \mathrm{dBm}$ at $2.5 \mathrm{GHz}$.

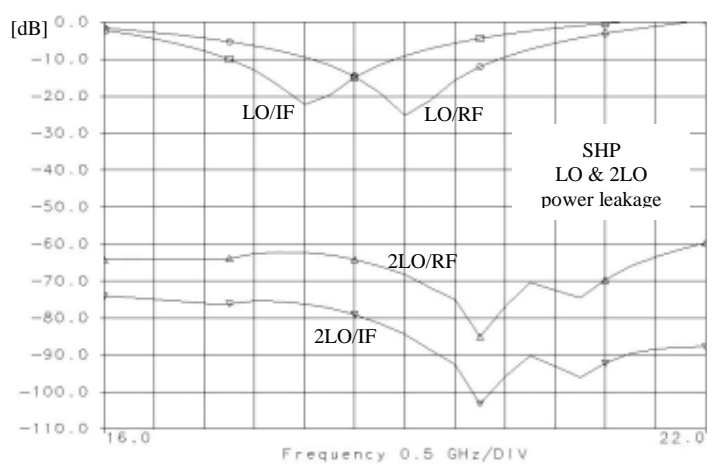

Fig. 4 - SHP mixer LO\&2LO power leakage on $\mathrm{RF} / \mathrm{IF}$ ports $\mathrm{LO}=+16 \mathrm{dBm}, \mathrm{IF}=-15 \mathrm{dBm} 2.5 \mathrm{GHz}$.

Since the mixer is sub-harmonic, the LO power is only partially suppressed on the broad-band of interest at the IF/RF ports, but it can be easily externally filtered due to the high distance from the IF and RF bands.

\section{B. Wilkinson Design}

The in-phase Wilkinson power splitter was realised in an almost-lumped elements structure in order to reduce the area occupancy with the aid of two open stubs instead of a very small capacitance not feasible due to process limitations. The simulated performance, 
displayed in figure 5 , give in the $17.25 \div 21.25 \mathrm{GHz}$ band, a three ports return loss and a ports isolation better than $-15 \mathrm{~dB}$ band with two in-phase way port transmission close to $-3.5 \mathrm{~dB}$.

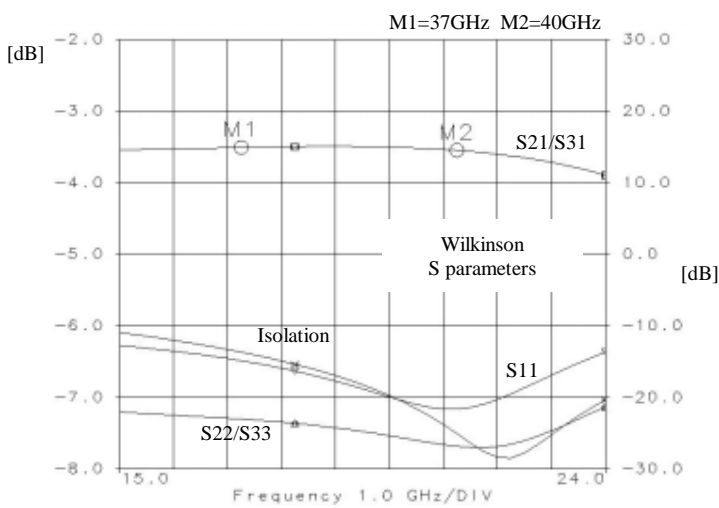

Fig.5 - S-parameters of Wilkinson power splitter

\section{Lange Coupler Design}

For the Lange coupler, we appropriately resized a process related structure, designed in the $\mathrm{N} 1$ metal PH25 process layer, the bottom capacitor layer, so to have lower metallization width and spacing for lower losses and better isolation properties, optimising it via the Agilent/Momentum electromagnetic simulator in the $37 \div 40 \mathrm{GHz}$ band. The S-parameters of the structure obtained, are shown in figure 6 , were it can be seen a flat $3.5 \mathrm{~dB}$ by channel loss and close to $-20 \mathrm{~dB}$ of ports isolation and return loss, in the RF band of interest.

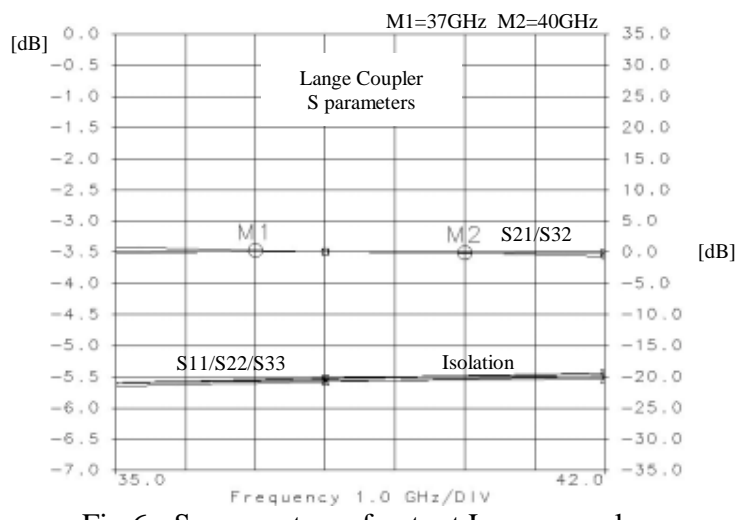

Fig.6 - S-parameters of output Lange coupler

\section{Buffer Stage Design}

The buffer stage was designed to operate close to saturation along with sufficient suppression of innergenerated harmonic components. The design efforts resulted in a single stage, unconditionally stable reactive feedback amplifier, compound of a $600 \mu \mathrm{m}$ HEMT, surrounded by an high-pass input and low-pass filtering output matching network. The simulations carried out at $\mathrm{V}_{\mathrm{ds}}=3 \mathrm{~V}, \mathrm{~V}_{\mathrm{gs}}=-0.25 \mathrm{~V}, \mathrm{I}_{\mathrm{ds}}=135 \mathrm{~mA}$, show a typical gain close to $7 \mathrm{~dB}$, a saturated output power close $20 \mathrm{dBm}$, and an harmonic suppression better than $60 \mathrm{~dB}$ in the band of interest, as can be seen in figure 7. The input operation level is $+12 \mathrm{dBm}$ so to provide a $+19 \mathrm{dBm}$ output level.

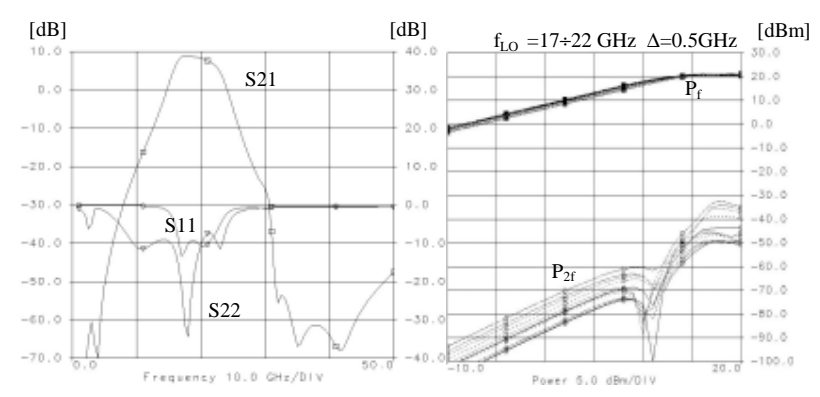

Fig.7 - Buffer S-parameters and fundamental and $2^{\text {nd }}$ harmonic Pout / Pin curves

\section{E. Final Mixer Design}

The previous shown designs were merged together to obtain the SHPIMR mixer with buffer desired design. Some optimisations on single designs were made. Input/output bond wires and microwave pads transition effects were taken into account and the behaviour versus process related elements variations was simulated. Due to the IF frequency of $2.5 \mathrm{GHz}$ relative to the RF one of $37 \div 40 \mathrm{GHz}$ and the sub-harmonic mixer type, the USB or LSB cases, are obtained, respectively, sweeping the LO in the $17.25 \div 18.75 \mathrm{GHz}$ or in the $19.75 \div 24.25 \mathrm{GHz}$ separate bands. For this reason we split the two cases, into two simulations. The SHPIMR mixer needs two quadrature IF signals, in this case simulated with an ideal $90^{\circ}$ hybrid. For an LO power of $+12 \mathrm{dBm}$ with a total IF power of $-15 \mathrm{dBm}$ at $2.5 \mathrm{GHz}$, at buffer bias $\mathrm{V}_{\mathrm{ds}}=3 \mathrm{~V} \mathrm{~V} \mathrm{~V}_{\mathrm{gs}}=-0.25 \mathrm{~V}$, the converted output performances, in the $37 \div 40 \mathrm{GHz}$ band, are shown in figure 8 and figure 9 for the USB case and in figure 10 and figure 11 for the LSB one. In the USB/LSB cases, conversion losses less than $11.5 \mathrm{~dB} / 12.5 \mathrm{~dB}$ with a $2 \mathrm{LO}$ to $\mathrm{RF} / \mathrm{IF}$ ports isolation best of $70 \mathrm{~dB}$ and an image rejection best of $20 \mathrm{~dB}$ can be obtained.

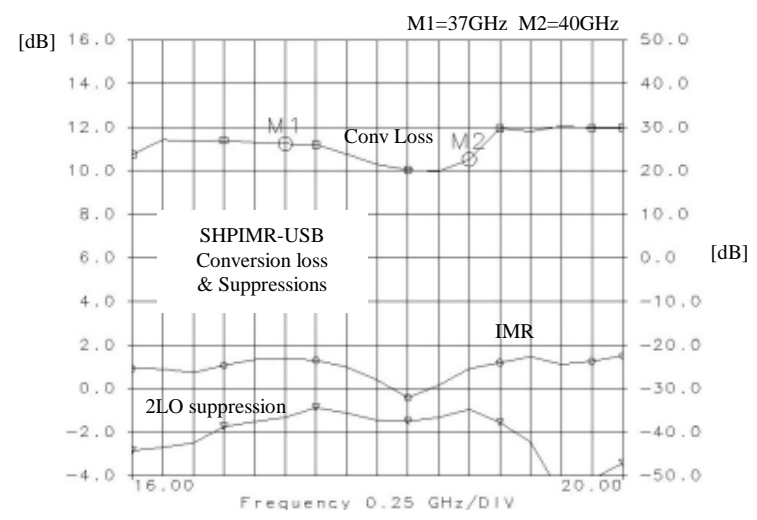

Fig. 8 - SHPIMR mixer USB conversion loss 


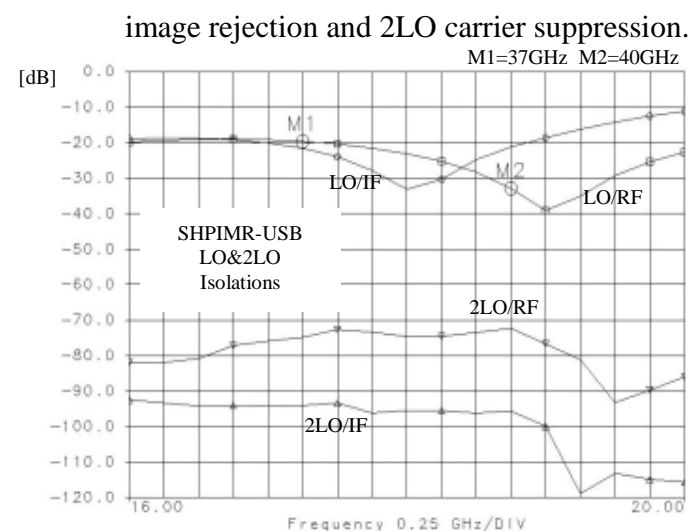

Fig.9 - SHPIMR mixer USB LO\&2LO to RF/IF ports isolations

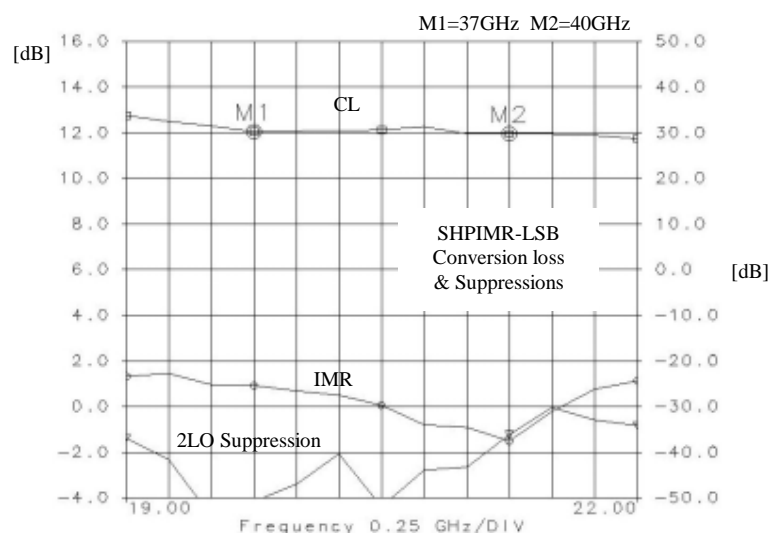

Fig. 10 - SHPIMR mixer LSB conversion loss image rejection and $2 \mathrm{LO}$ carrier suppression.

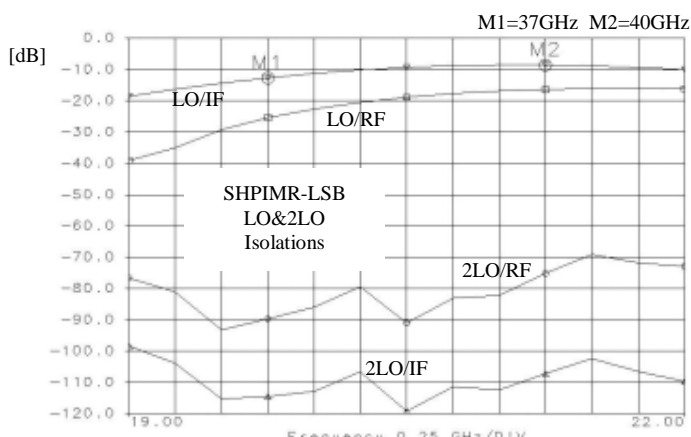

Fig. 11 - SHPIMR mixer LSB LO\&2LO to RF/IF ports isolations

The mixer simulated power performances and $\mathrm{IM}_{3}$ extrapolated $\left(\mathrm{IP}_{3}-\mathrm{P}_{1 \mathrm{~dB}}=8 \mathrm{~dB}\right)$ values, are in Table $\mathrm{I}$ summarised for the USB and LSB cases at $\mathrm{P}_{\mathrm{LO}}=+12 \mathrm{dBm}, \mathrm{IF}=2.5 \mathrm{GHz}, \mathrm{V}_{\mathrm{ds}}=3 \mathrm{~V} \mathrm{~V}_{\mathrm{gs}}=-0.25 \mathrm{~V}$.

\begin{tabular}{|c|c|c|c|c|c|c|c|c|}
\hline$f_{\mathrm{RF}}$ \\
\hline$[\mathrm{GHz}]$
\end{tabular} \begin{tabular}{|c|c|c|c|c|c|}
\hline $\mathrm{f}_{\mathrm{LO}-}$ & $\mathrm{P}_{\mathrm{ldB}}$ & $\mathrm{IP}_{3}$ & $\mathrm{IM}_{3}{ }^{*}$ \\
\hline$[\mathrm{GHz}]$ & {$[\mathrm{dBm}]$} & {$[\mathrm{dBm}]$} & {$[\mathrm{dBc}]$} \\
\hline
\end{tabular}

\begin{tabular}{|c|c|c|c|c|c|c|c|c|}
\hline 37 & 17,25 & $-6,0$ & 2,0 & $-62,0$ & 19,75 & $-5,2$ & 2,8 & $-63,6$ \\
\hline 39,5 & 18,50 & $-6,9$ & 1,1 & $-60,2$ & 20,00 & $-5,2$ & 2,8 & $-63,6$ \\
\hline 40 & 18,75 & $-6,5$ & 1,5 & $-61,0$ & 21,25 & $-5,1$ & 2,9 & $-63,8$ \\
\hline
\end{tabular}

Table I - P1dB\&IP3 performances. IV. MIXER LAYOUT

The final SHPIMR mixer with LO buffer stage MMIC layout is shown in figure 12. Two IF quadrature mirrored ports, LO input and RF output ports, plus drain-gate DC pads are present. The signal ports were designed in microwave probe pads configuration, in order to permit on-wafer characterisations. A multiproject tile set of masks was designed in order to have two design versions (driven by the e.m simulations) to increase the probability of first run final goal; a mixer structure with no buffer and a test patterns with buffer stage, Wilkinson divider and Lange coupler designs alone, in a microwave probe pads environment, were put to permits a full RF characterisation for a back simulations, if a second process run will return necessary.

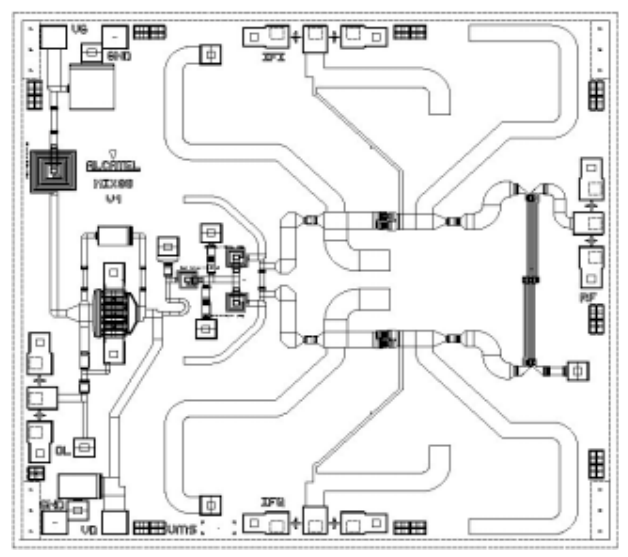

Fig.12 - SHPIMR with LO buffer mixer layout $2.37 \times 2.31 \mathrm{~mm}^{2}$

\section{CONCLUSIONS}

A compact size SHPIMR MMIC mixer for $37 \div 40 \mathrm{GHz}$ band operation has been designed. The wafer process is under development; the MMIC will be used in a $38 \mathrm{GHz}$ transmitter module developed in a cooperation program joining Alcatel Italia, Thomson-CSF and UMS efforts. The design goals performances joined with the careful design approach and the deep foundry collaboration are a good omen for the final MMIC success. 


\section{REFERENCES}

[1] S.A. Maas, Microwave Mixers, Second Edition, Artech House - Boston $\circ$ London, 1993

[2] E.R. Carlson, M.V. Schneider, T.F. McMaster "Subharmonically Pumped Millimeter-Wave Mixers", IEEE 1978 Trans. Microwave Theory Tech., vol. MTT26, pp.706-715, October 1978.

[3] PH25 MMIC Foundry Design Manual, Version 2.0, United Monolithic Semiconductors 